\title{
Solving Maxwell eigenvalue problems for accelerating cavities
}

\author{
Peter Arbenz* and Roman Geus ${ }^{\dagger}$ \\ Swiss Federal Institute of Technology (ETH), Institute of Scientific Computing, CH-8092 Zurich, Switzerland \\ Stefan Adam ${ }^{*}$ \\ Paul-Scherrer-Institute, CH-5232 Villigen, Switzerland
}

(Received 24 October 2000; published 26 February 2001)

\begin{abstract}
We investigate algorithms for computing steady state electromagnetic waves in cavities. The Maxwell equations for the strength of the electric field are solved by a mixed method with quadratic finite edge (Nédélec) elements for the field values and corresponding node-based finite elements for the Lagrange multiplier. This approach avoids so-called spurious modes which are introduced if the divergence-free condition for the electric field is not treated properly. To compute a few of the smallest positive eigenvalues and corresponding eigenmodes of the resulting large sparse matrix eigenvalue problems, two algorithms have been used: the implicitly restarted Lanczos algorithm and the Jacobi-Davidson algorithm, both with shift-and-invert spectral transformation. Two-level hierarchical basis preconditioners have been employed for the iterative solution of the resulting systems of equations.
\end{abstract}

DOI: 10.1103/PhysRevSTAB.4.022001

PACS numbers: $29.20 . \mathrm{Hm}, 41.20 . \mathrm{Jb}, 02.60 . \mathrm{Dc}$

\section{INTRODUCTION}

The common way to produce the accelerating electromagnetic fields in cyclic accelerators is to excite standing waves in accelerating cavities. The mathematical model for these high frequency electromagnetic fields is the eigenvalue problem solving the Maxwell equations in a bounded volume [1].

Usually, the eigenfield corresponding to the fundamental mode of the cavity is used as the accelerating field. A few modes of higher order have to be analyzed as well because these modes can be excited due to higher harmonic components contained in the rf power fed into the cavity and through interactions between the accelerated particles and the electromagnetic field. The rf engineer designing such an accelerating cavity therefore needs a tool to compute the fundamental and about 10 to 20 of the following eigenfrequencies together with the corresponding electromagnetic eigenfields. The most interesting quantities besides the eigenfrequencies are local maxima of the eigenmodes as well as the fields on the surface that induce heat in the metallic boundary and determine the power loss from surface currents.

The finite element formulation for the solution of this problem has been chosen for its high flexibility for the geometric modeling, in particular, with strong variations of the scale of structural details.

For the solution of Maxwell eigenvalue problems, Kikuchi [2] suggested a mixed formulation method based

\footnotetext{
*Electronic address: arbenz@inf.ethz.ch

${ }^{\dagger}$ Electronic address: geus@inf.ethz.ch

*Electronic address: Stefan.Adam@psi.ch
}

on Nédélec's edge elements [3,4] for the electric field and node-based elements for the Lagrange multiplier. In this way, the computed approximate eigenmodes are naturally split into (physically meaningless) curl-free and (in a discrete sense) divergence-free modes. Spurious modes cannot appear. In the engineering literature, the advantages and disadvantages of Nédélec's edge elements versus other finite element formulations are discussed intensely [5,6]. In the late 1970s, Weiland $[7,8]$ invented the so-called finite integration technique (FIT) that completely avoids the problem of spurious modes. However, as it is based on a finite difference scheme, it is less flexible in modeling complex resonator geometries.

By using numerical experiments reported in [9], we identified Sorensen's implicitly restarted Lanczos (IRL) algorithm [10] and the Jacobi-Davidson (JD) algorithm [11] to be the algorithms best suited to solve the large sparse eigenvalue problems that arise with the above finite element discretizations. They are to be preferred to subspace iteration [12] or the block Lanczos algorithm [13].

In this paper we report on our continuing investigations on eigensolvers and their preconditioning. In contrast to [9] we conduct our experiments on a domain that approximates the new cavity design for the $590 \mathrm{MeV}$ ring cyclotron at the Paul Scherrer Institute (PSI) in Villigen, Switzerland; see Fig. 1, right-hand side.

The outline of the paper is as follows. In Sec. II we will give the mathematical formulation of the problem. In Sec. III the Nédélec finite element approach for tetrahedral meshes is looked at from a linear algebra point of view. In Secs. IV-VI we detail how we solve the large eigenvalue problems and, in particular, solve the systems of equations that are introduced by the spectral transformation. In Sec. VII we compare the performance of several variants in solving the model problem. 


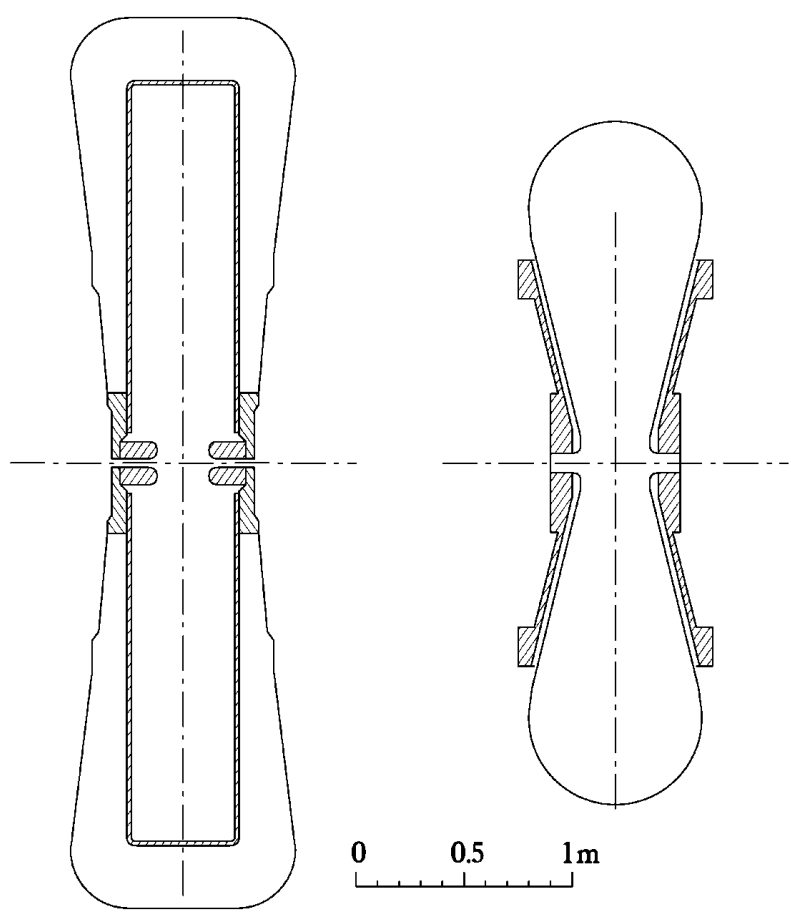

FIG. 1. Cross section of two different designs for accelerating cavities. On the left, the original design that is presently in operation; on the right, the future design. The plane of these two cross sections is perpendicular to the accelerator midplane and tangential to the orbit of the accelerated particles. Both cavities extend prismatically in the radial direction over approximately $3.3 \mathrm{~m}$.

\section{STATEMENT OF THE PROBLEM}

Without changing the basic structure of the problem one can assume that the metallic surfaces are perfectly conducting and that the inside volume of the cavity $\Omega$ is all in vacuum. The electromagnetic field in the cavity is described by the Maxwell equations [1]. After separation of time and space and after elimination of the magnetic field intensity the differential equations

$$
\begin{gathered}
\operatorname{curl} \operatorname{curl} \mathbf{e}(\mathbf{x})=\lambda \mathbf{e}(\mathbf{x}), \quad \mathbf{x} \in \Omega, \quad \lambda:=\omega^{2} / c^{2}, \\
\operatorname{dive}(\mathbf{x})=0, \quad \mathbf{x} \in \Omega, \\
\mathbf{n} \times \mathbf{e}=0, \quad \mathbf{x} \in \Gamma:=\partial \Omega,
\end{gathered}
$$

are obtained for the electric field intensity e. Equations (1) have solutions only for certain values $\omega$ called eigenfrequencies of the electromagnetic oscillations of the resonator. These solutions are called eigenmodes.

The eigenvalue problem (1) can be solved analytically for a few particular domains, e.g., when $\Omega$ is a rectangular box or a right circular cylinder [1]. We intend to solve (1) in more complicated domains $\Omega$ by the finite element method. To that end we have to write the eigenvalue problem in variational form. There are a number of ways to do so. The mixed formulation proposed by Kikuchi [2]
Find $(\lambda, \mathbf{e}, p) \in \mathbb{R} \times H_{0}(\mathbf{c u r l} ; \Omega) \times H_{0}^{1}(\Omega)$

$$
\text { such that } \mathbf{e} \neq \mathbf{0} \text { and }
$$

(a) $(\operatorname{curl}$ e, curl $\Psi)+(\operatorname{grad} p, \boldsymbol{\Psi})$

$$
=\lambda(\mathbf{e}, \boldsymbol{\Psi}), \quad \forall \boldsymbol{\Psi} \in H_{0}(\operatorname{curl} ; \Omega)
$$

(b) $(\mathbf{e}, \operatorname{grad} q)=0, \quad \forall q \in H_{0}^{1}(\Omega)$

is useful as it leads to a mixed finite element formulation that is free of spurious modes $[5,6]$. Here, $(\mathbf{u}, \mathbf{v})=\int_{\Omega} \mathbf{u}(\mathbf{x}) \cdot \mathbf{v}(\mathbf{x}) d \mathbf{x}$ denotes the usual inner product in $L^{2}(\Omega)^{3}$, the space of three-vector valued square integrable functions on $\Omega$. The well-known Sobolev space [14]

$$
\begin{aligned}
H_{0}^{1}(\Omega):=\left\{q \in L^{2}(\Omega) \mid \operatorname{grad} q \in L^{2}(\Omega)^{3},\right. & \\
& q=0 \text { on } \Gamma\}
\end{aligned}
$$

and the Hilbert space $[15,16]$

$$
\begin{aligned}
H_{0}(\mathbf{c u r l} ; \Omega):=\left\{\mathbf{v} \in L^{2}(\Omega)^{3} \mid \operatorname{curl} \mathbf{v}\right. & \in L^{2}(\Omega)^{3}, \\
\mathbf{v} & \times \mathbf{n}=\mathbf{0} \text { on } \Gamma\}
\end{aligned}
$$

are used in the formulation (2).

It is easy to see that $p=0$ for all solutions of (2) (see [2]), and that there is a one-to-one correspondence between the solutions $(\lambda, \mathbf{e})$ of (1) and the solutions $(\lambda, \mathbf{e}, 0)$ of (2).

In the next section we investigate the finite element discretization of $H_{0}(\mathbf{c u r l} ; \Omega)$ and $H_{0}^{1}(\Omega)$ by Nédélec edge elements and Lagrange node elements, respectively. If also $H_{0}(\mathbf{c u r l} ; \Omega)$ is approximated by Lagrange elements the eigenvalue zero will not be confined in the neighborhood of zero and can cause spectral pollution.

\section{FINITE ELEMENT DISCRETIZATIONS}

In this section we discuss finite element approximations of the mixed formulation (2). We assume that $\Omega$ is an open, bounded domain in $\mathbb{R}^{3}$ with a polyhedral boundary $\Gamma$. The domain is triangulated by tetrahedrons.

We replace $H_{0}(\mathbf{c u r l} ; \Omega)$ and $H_{0}^{1}(\Omega)$ by finite dimensional subspaces $V_{h}$ and $W_{h}$, respectively, to obtain

Find $\left(\lambda_{h}, \mathbf{e}_{h}, p_{h}\right) \in \mathbb{R} \times V_{h} W_{h} \quad$ such that $\mathbf{e}_{h} \neq \mathbf{0}$ and

(a) $\left(\operatorname{curl} \mathbf{e}_{h}, \operatorname{curl} \boldsymbol{\Psi}_{h}\right)+\left(\operatorname{grad} p_{h}, \boldsymbol{\Psi}_{h}\right)=\lambda_{h}\left(\mathbf{e}_{h}, \boldsymbol{\Psi}_{h}\right)$,

$$
\forall \boldsymbol{\Psi}_{h} \in V_{h},
$$

(b) $\left(\mathbf{e}_{h}, \operatorname{grad} q_{h}\right)=0, \quad \forall q_{h} \in W_{h}$.

The space $W_{h} \subset H_{0}^{1}(\Omega)$ is the well-known Lagrange (or node-based) finite element space [14],

$$
W_{h}^{(k)}:=\left\{\mathbf{v} \in W|\mathbf{v}|_{\Omega_{e}} \in\left[P_{k}\left(\Omega_{e}\right)\right]^{3}\right\},
$$

where $P_{k}\left(\Omega_{e}\right)$ is the space of polynomials in the tetrahedral subdomains $\Omega_{e}$ of degree $\leq k$. In this paper we exclusively deal with $k=2$. We work with a hierarchical basis and write 


$$
W_{h}^{(2)}=W_{h}^{(1)} \oplus \bar{W}_{h}^{(2)}
$$

The natural basis of $W_{h}^{(1)}$ is formed by the piecewise linear polynomials that are 1 in one node (vertex) $k$ and 0 in all others. Thus, $m_{1}:=\operatorname{dim} W_{h}^{(1)}$ equals the number of interior vertices of the triangulation. The basis of $\bar{W}_{h}^{(2)}$ consists of those piecewise quadratic polynomials that are 1 in the midpoint of one edge and 0 in the midpoints of all other edges and in all vertices. So, $m_{2}=m-m_{1}=$ $\operatorname{dim} \bar{W}_{h}^{(2)}$ is equal to the number of interior edges of the triangulation.

The space $V_{h}$ is made up of the $H(\mathbf{c u r l} ; \Omega)$ conforming finite elements of order $k \leq 2$ proposed by Nédélec [4,16-18]. We again use a hierarchical basis and write

$$
V_{h}^{(2)}=V_{h}^{(1)} \oplus \bar{V}_{h}^{(2)}
$$

The basis of $V_{h}^{(1)}$ consists of piecewise linear vector valued functions with tangential component equal to 1 on one interior edge and equal to 0 on all other edges. Therefore, $n_{1}:=\operatorname{dim} V_{h}^{(1)}=m_{2}$. The basis of $\bar{V}_{h}^{(2)}$ consists of two sets of functions. The first set is made up of piecewise quadratic polynomials that have a linearly varying tangential component along one interior edge and are equal to zero on all other edges. Each of these basis functions is curl free and equals the gradient of the basis functions of $\bar{W}_{h}^{(2)}$ corresponding to the same edge. The second set of basis functions of $\bar{V}_{h}^{(2)}$ is made up of piecewise quadratic polynomials that have their degrees of freedom on the element faces. There are two basis functions per face. They are defined by the two zeroth moments of the tangential components on the face. So, $n_{2}=n-n_{1}=\operatorname{dim} \bar{V}_{h}^{(2)}$ is equal to the number of interior edges plus twice the number of interior faces of the triangulation.

Let $\left\{\boldsymbol{\Phi}_{i}\right\}_{i=1}^{n}$ be a basis of $V_{h}^{(2)}$ and $\left\{\varphi_{l}\right\}_{l=1}^{m}$ be a basis of $W_{h}^{(2)}$. Then (3) is equivalent to the matrix eigenvalue problem

$$
A \mathbf{x}=\lambda M \mathbf{x}, \quad C^{T} \mathbf{x}=\mathbf{0},
$$

where $A$ and $M$ are $n \times n$ and $C$ is $n \times m$. We partition the matrices $A, M$, and $C$ according to the hierarchical bases,

$$
\begin{gathered}
A=\left[\begin{array}{ll}
A_{11} & A_{12} \\
A_{21} & A_{22}
\end{array}\right], \quad M=\left[\begin{array}{ll}
M_{11} & M_{12} \\
M_{21} & M_{22}
\end{array}\right], \\
C=\left[\begin{array}{ll}
C_{11} & C_{12} \\
C_{21} & C_{22}
\end{array}\right],
\end{gathered}
$$

with $A_{i j}, B_{i j} \in \mathbb{R}^{n_{i} \times n_{j}}$ and $C_{i j} \in \mathbb{R}^{n_{i} \times m_{j}}$. The $(1,1)$ blocks correspond to the bilinear forms involving basis functions in $V_{h}^{(1)}$ and $W_{h}^{(1)}$, respectively.

Formally, spaces consisting of common Lagrange finite elements could be chosen for $V_{h}$ and $W_{h}$ in (3). However, Nédélec's edge elements have the crucial advantage over Lagrange finite elements that [16]

$$
\operatorname{grad} W_{h}^{(2)}=\left\{\mathbf{v}_{h} \in V_{h}^{(2)} \mid \operatorname{curl} \mathbf{v}_{h}=\mathbf{0}\right\}
$$

Similar to the continuous case, we can decompose any $\mathbf{v}_{h} \in V_{h}^{(2)}$ in the form $\mathbf{v}_{h}=\left(\mathbf{v}_{h}-\operatorname{grad} q_{h}\right)+$ $\operatorname{grad} q_{h}=: \mathbf{w}_{h}+\operatorname{grad} q_{h}$, where $q_{h} \in W_{h}^{(2)}$ is defined by

$$
\left(\operatorname{grad} q_{h}, \operatorname{grad} \varphi\right)=\left(\mathbf{v}_{h}, \operatorname{grad} \varphi\right), \quad \forall \varphi \in W_{h}^{(2)} .
$$

Then, $\mathbf{v}_{h}=\mathbf{w}_{h}+\operatorname{grad} q_{h}$ splits $V_{h}^{(2)}$-in a discrete sense - into a divergence-free part and a curl-free part. We write this so-called Helmholtz decomposition as

$$
V_{h}^{(2)}=W_{0, h}^{(2)} \oplus \operatorname{grad} W_{h}^{(2)} \text {. }
$$

For $\mathbf{w}_{h} \in W_{0, h}^{(2)} \backslash\{\mathbf{0}\}$ we have $\left\|\mathbf{c u r l} \mathbf{w}_{h}\right\|_{0, \Omega}>0$. Notice that $W_{0, h}^{(2)}$ is not contained in $W_{0}$. We therefore cannot expect monotonic convergence of the eigenvalues. Equation (9) corresponds to the "nonpollution condition" for the conforming finite element approximation of the model problem by Gruber and Rappaz [19]. Therefore, the infinitely multiple eigenvalue zero of curl curl is not spread over the real axis but remains confined in the origin.

In order that $\mathbf{u}_{h}=\sum_{j=1}^{n} \xi_{j} \boldsymbol{\Phi}_{j}$ is in $W_{0, h}^{(2)}$, we must have

$$
\begin{aligned}
\left(\operatorname{grad} \varphi_{l}, \mathbf{u}_{h}\right)=\sum_{j=1}^{n}\left(\operatorname{grad} \varphi_{l}, \boldsymbol{\Phi}_{j}\right) \xi_{j} & =0, \\
1 & \leq l \leq m,
\end{aligned}
$$

or, equivalently,

$$
C^{T} \mathbf{x}=\mathbf{0}, \quad \mathbf{x}=\left(\xi_{1}, \ldots, \xi_{n}\right)^{T}
$$

which is the second equation in (7). Because of (9) we can write $\operatorname{grad} \varphi_{l}=\sum_{j=1}^{n} \eta_{j l} \boldsymbol{\Phi}_{j}$. Therefore,

$$
\left(\boldsymbol{\Phi}_{i}, \operatorname{grad} \varphi_{l}\right)=\sum_{j=1}^{n}\left(\boldsymbol{\Phi}_{i}, \boldsymbol{\Phi}_{j}\right) \eta_{j l}, \quad 1 \leq l \leq m
$$

or

$$
C=M\left[\mathbf{y}_{1}, \ldots, \mathbf{y}_{m}\right]=: M Y, \quad \mathbf{y}_{l}^{T}=\left(\eta_{1 l}, \ldots, \eta_{n l}\right) .
$$

In a similar way one obtains

$$
H:=C^{T} Y=Y^{T} M Y, \quad h_{k l}=\left(\operatorname{grad} \varphi_{k}, \operatorname{grad} \varphi_{l}\right) .
$$

$H$ is the system matrix that is obtained when solving the Poisson equation with the Lagrange finite elements $W_{h}^{(2)}$.

Notice that $Y$ is very sparse. We have already mentioned that the gradient of a basis function $\varphi_{k} \in \bar{W}_{h}^{(2)}$ is an element of the first set of basis functions of $\bar{V}_{h}^{(2)}$. So, $m_{1}$ rows of $Y$ have a single entry 1 . The gradient of the piecewise linear basis function corresponding to vertex $k$, say, is a linear combination (with coefficients \pm 1 ) of the basis functions of $V_{h}^{(1)}$ whose corresponding edge has vertex $k$ as one of its end points. 
Equation (11) means that $C^{T} \mathbf{x}=\mathbf{0}$ is equivalent to requiring $\mathbf{x}$ to be $M$ orthogonal to the eigenspace $\mathcal{N}(A)=$ $\mathcal{R}(Y)$ corresponding to the eigenvalue 0 . Thus, the solutions of (7) are precisely the eigenpairs of

$$
A \mathbf{x}=\lambda M \mathbf{x}
$$

corresponding to the positive eigenvalues. We could therefore compute the desired eigenpairs $\left(\lambda_{j}, \mathbf{x}_{j}\right)$ of (7) by means of (12) alone. The computed eigenvectors corresponding to positive eigenvalues would automatically satisfy the constraint $C^{T} \mathbf{x}_{j}=\mathbf{0}$. This is actually done if the linear systems of the form $(A-\sigma M) \mathbf{x}=M \mathbf{y}$ that appear in the eigensolver can be solved by direct methods. Numerical experiments showed, however, that the highdimensional zero eigenspace has a negative effect on the convergence rates if preconditioned iterative solvers have to be used.

\section{SOLVING THE MATRIX EIGENVALUE PROBLEM}

The classic method for computing a few selected eigenpairs of $A \mathbf{x}=\lambda M \mathbf{x}$ is subspace iteration [12,20]. Its linear convergence rate is, however, too low to be competitive with modern algorithms. The Lanczos algorithm in turn converges exponentially $[12,13]$. Its main drawback is that it requires memory space proportional to the number of iteration steps that have to be made until convergence. These huge memory requirements can cause the Lanczos algorithm to stop prematurely before convergence has taken place. These well-known facts have been verified for the rectangular cavity [9]. The algorithms that successfully solved all problem sizes were the implicitly restarted Lanczos algorithm [10,21,22] and the Jacobi-Davidson algorithm [11,23,24]. Like Lanczos, these algorithms converge superlinearly but, like subspace iteration, their memory requirements are determined by the order $n$ of the problem times a small multiple of the number $p$ of the desired eigenvalues.

In this study we use the implicitly restarted Lanczos algorithm and the Jacobi-Davidson algorithm as described in [9] to solve the matrix eigenvalue problem (7) that we write in the form

$$
M^{-1} A \mathbf{x}=\lambda \mathbf{x}, \quad C^{T} \mathbf{x}=Y^{T} M \mathbf{x}=\mathbf{0} .
$$

Note that $M^{-1} A$ is symmetric (self-adjoint) with respect to the inner product $\langle\mathbf{x}, \mathbf{y}\rangle=\mathbf{x}^{T} M \mathbf{y}$. Admissible vectors $\mathbf{x}$ are orthogonal to the null space $\mathcal{N}(A)=\mathcal{R}(Y)$ of $A$. Thus, $M^{-1} A$ is positive definite as an operator from $\mathcal{R}(Y)^{\perp_{M}}$ onto itself. The lowest part of its spectrum is desired.

\section{A. Spectral transformations}

When computing a few, say $p$, eigenvalues of (13) closest to a number $\tau$ it is essential to apply a spectral transformation to get a reasonable speed of convergence $[13,25-27]$. With the shift-and-invert spectral transformation (13) becomes

$$
\begin{gathered}
(A-\sigma M)^{-1} M \mathbf{x}=\mu \mathbf{x}, \quad C^{T} \mathbf{x}=\mathbf{0}, \\
\mu=\frac{1}{\lambda-\sigma} .
\end{gathered}
$$

The shift $\sigma$ is chosen close to $\tau$. Of course, $A-\sigma M$ must be nonsingular. The spectral transformation leaves the eigenvectors unchanged. The eigenvalues of (13) close to the shift $\sigma$ become the largest absolute of (14). In addition they are relatively well separated, which improves the speed of convergence of the eigensolvers [12]. The cost of the improved convergence rate is solving a system of equations of the form

$$
(A-\sigma M) \mathbf{x}=M \mathbf{y}, \quad C^{T} \mathbf{x}=\mathbf{0},
$$

in each iteration step of the eigensolver. We assume that $A$ and $M$ are so big that they cannot be factored such that (15) has to be solved iteratively. In the subsequent sections we are going to discuss how to do this and, in particular, how to precondition (15).

\section{B. Implicitly restarted Lanczos algorithm}

To overcome the memory consumption of the Lanczos algorithm it has to be restarted in some way. Sorensen [10] suggested an elegant way to do so. He showed how to extract a Krylov subspace with given spectral properties from the actual trial space (also Krylov subspace). These ideas that apply to the Arnoldi algorithm for nonsymmetric eigenvalue problems as well are implemented in the Fortran subroutine package ARPACK [28].

The Lanczos iteration process with shift-and-invert spectral transformation is executed until $j=p+k$, where $k$ is some positive integer. We used $k=p$ as suggested in [28]. Complete reorthogonalization to guarantee stability is feasible as, by assumption, $p+k$ is not big. If $j=p+k$ then the dimension of the trial space is reduced to $p$ with a procedure related to the implicit shifted QR algorithm [12]. The shifts are chosen to be the $k$ eigenvalues of the tridiagonal matrix produced by the Lanczos iteration process that are furthest away from the target $\tau$. As the $p$ dimensional subspace left after the above procedure is again a Krylov subspace, the Lanczos iteration process can be restarted.

In the IRL, the system of equations (15) has to be solved to high accuracy as otherwise the Lanczos relation among the basis vectors does not hold true. We followed the recommendation in [28] to set the convergence tolerance tol for the systems of equation 100 times smaller than the accuracy $\varepsilon$ required for the eigenpairs; see Sec. VII.

Besides the storage for the matrices $A$ and $M$, the memory requirements of IRL are essentially the space needed to store $2 n(p+k)$ floating point variables. 


\section{Jacobi-Davidson algorithm}

The Jacobi-Davidson algorithm has been introduced by Sleijpen and van der Vorst [11] for solving arbitrary generalized eigenvalue problems. We have adapted their algorithm JDQZ to the generalized eigenvalue problem with symmetric $A$ and symmetric positive definite $M$ [9].

The Jacobi-Davidson algorithm is not a Krylov subspace method. The trial space spanned by the $M$-orthogonal columns of $V_{j} \in \mathbb{R}^{n \times j}$ used in JD is extended by means of the solution $\mathbf{t}$ of the correction equation

$$
\begin{aligned}
\left(I-\tilde{W}_{k} \tilde{Q}_{k}^{T}\right)(A-\tilde{\lambda} M)\left(I-\tilde{Q}_{k} \tilde{W}_{k}^{T}\right) \mathbf{t}=-\mathbf{r}, \\
\left(I-\tilde{Q}_{k} \tilde{W}_{k}^{T}\right) \mathbf{t}=\mathbf{t},
\end{aligned}
$$

where $\tilde{Q}_{k}=\left[\mathbf{q}_{1}, \ldots, \mathbf{q}_{k}, \tilde{\mathbf{q}}\right]$ and $\tilde{W}_{k}=M \tilde{Q}_{k}$. Here, $(\tilde{\lambda}, \tilde{\mathbf{q}})$ is a Ritz pair in the actual trial space and $\mathbf{q}_{1}, \ldots, \mathbf{q}_{k}$ are the already computed eigenvectors. Initially, $k=0$. Notice that $\left(I-\tilde{W}_{k} \tilde{Q}_{k}^{T}\right) \mathbf{r}=\mathbf{r}$ such that $(16)$ is consistent if $A-\tilde{\lambda} M$ is invertible. The solution $\mathbf{t}$ of (16) is $M$ orthogonalized against the columns of $V_{j}$ and appended to $V_{j}$ to obtain $V_{j+1}$.

If $j=j_{\max }$, the dimension of the trial space is reduced to $j=j_{\min } \geq p$ by choosing the $j_{\min }$ most desirable Ritz vectors from $\mathcal{R}\left(V_{j_{\max }}\right)$.

If (16) is solved exactly, one step of the algorithm turns out to be one step of the Rayleigh quotient iteration [11] which converges cubicly for symmetric eigenvalue problems [12]. If (16) is solved only approximately, this high convergence rate gets lost. There is a trade-off between speed of convergence and the amount of work one is willing to spend for solving (16).

If Eq. (16) is solved approximately by a Krylov subspace method, a good preconditioner has to be employed to get a satisfactory convergence rate. Notice that the matrix in (16) maps on the orthogonal complement of $\tilde{Q}_{k}$ instead of $\tilde{W}_{k}$. Therefore, the preconditioner also has the duty to map the solutions in the correct subspace. Similar to Fokkema et al. [24], we use a preconditioner of the form

$$
\left(I-\tilde{W}_{k} \tilde{Q}_{k}^{T}\right) K\left(I-\tilde{Q}_{k} \tilde{W}_{k}^{T}\right): \mathcal{R}\left(\tilde{W}_{k}\right)^{\perp} \rightarrow \mathcal{R}\left(\tilde{Q}_{k}\right)^{\perp},
$$

where the symmetric matrix $K$ is a good and easily invertible approximation of $A-\tilde{\lambda} M$. It is straightforward to show that the inverse of this mapping is given by

$$
\left[I-\tilde{Y}_{k}\left(\tilde{W}_{k}^{T} \tilde{Y}_{k}\right)^{-1} \tilde{W}_{k}^{T}\right] K^{-1}\left(I-\tilde{W}_{k} \tilde{Q}_{k}^{T}\right),
$$

where $\tilde{Y}_{k}=K^{-1} \tilde{W}_{k}$. The preconditioned equation (16) then becomes

$$
\begin{aligned}
& {\left[I-\tilde{Y}_{k}\left(\tilde{W}_{k}^{T} \tilde{Y}_{k}\right)^{-1} \tilde{W}_{k}^{T}\right] K^{-1}\left(I-\tilde{W}_{k} \tilde{Q}_{k}^{T}\right)(A-\tilde{\lambda} M) \mathbf{t} } \\
& \quad=\left[I-\tilde{Y}_{k}\left(\tilde{W}_{k}^{T} \tilde{Y}_{k}\right)^{-1} \tilde{W}_{k}^{T}\right] K^{-1}(A-\tilde{\lambda} M) \mathbf{t} \\
& \quad=-\left[I-\tilde{Y}_{k}\left(\tilde{W}_{k}^{T} \tilde{Y}_{k}\right)^{-1} \tilde{W}_{k}^{t}\right] K^{-1} \mathbf{r} \\
& \tilde{W}_{k}^{T} \mathbf{t}=0 .
\end{aligned}
$$

Memory space is needed for the blocks $\tilde{Q}_{k}, \tilde{W}_{k}, \tilde{Y}_{k}$, and $V$. Thus, besides the storage for the matrices $A-\sigma M$ and $M,\left(3 p+j_{\max }\right) n$ memory locations are needed.

\section{SOLVING THE CONSTRAINED SYSTEM OF EQUATION: THE STRAIGHTFORWARD APPROACH}

We investigate two methods to solve (15). In the straightforward approach of this section we apply the conjugate gradient method forcing the iterates into $\mathcal{R}(C)^{\perp}=\mathcal{R}(Y)^{\perp_{M}}$. In the second approach of Sec. VI we treat (15) as an augmented system of order $n+m$.

We exploit that

$$
M^{-1}(A-\sigma M): \mathcal{R}(Y)^{\perp_{M}} \rightarrow \mathcal{R}(Y)^{\perp_{M}}
$$

is symmetric positive definite and apply the preconditioned conjugate gradient (pcg) method [29] to solve $M^{-1}(A-$ $\sigma M) \mathbf{x}=\mathbf{y}$. Applying the preconditioner $M^{-1}$ amounts to approximately solving

$$
(A-\sigma M) \mathbf{x}=M \mathbf{y} .
$$

Notice that both $M$ and $A-\sigma M$ map $\mathcal{R}(Y)^{\perp_{M}}$ one to one onto $\mathcal{R}(Y)^{\perp}$. Two problems arise if (20) is solved as it stands. First, $(A-\sigma M)$ as a map of the whole $n$ space is indefinite and is bound to slow down convergence [30]. Second, if (20) is not solved to high accuracy the approximate solution will fail to satisfy the constraints in (15). By choosing the preconditioner

$$
M^{-1} P_{\mathcal{R}(Y)^{\perp} M}^{T}:=M^{-1}\left(I-C H^{-1} Y^{T}\right),
$$

the pcg iterates stay in the correct space. In the JacobiDavidson algorithm the approximate solution $\mathbf{t}$ of (16) is additionally forced to be orthogonal to the Ritz vector $\tilde{\mathbf{q}}$ and to the previously computed eigenvectors.

Here, we are mainly interested in two-level preconditioners induced by the hierarchical finite element basis. Proceeding as Bank [31], we approximate $A, M$, and $C$ partitioned as in (8) by the block diagonal

$$
\begin{gathered}
A_{1}=\left[\begin{array}{cc}
A_{11} & O \\
O & A_{22}
\end{array}\right], \quad M_{1}=\left[\begin{array}{cc}
M_{11} & O \\
O & M_{22}
\end{array}\right], \\
C_{1}=\left[\begin{array}{cc}
C_{11} & O \\
O & C_{22}
\end{array}\right] .
\end{gathered}
$$

$A_{1}, M_{1}$, and $C_{1}$ have similar properties as $A, M$, and $C$; in particular, $A_{1} Y=O$ and $C_{1}=M_{1} Y$. Further, $A_{1}-$ $\sigma_{1} M_{1}$ maps $\mathcal{R}(Y)^{\perp_{M_{1}}}$ onto $\mathcal{R}(Y)^{\perp}$. Now we define a preconditioner

$$
M^{-1}\left(A_{1}-\sigma_{1} M_{1}\right) P_{\mathcal{R}(Y)^{\perp_{1}}}: \mathcal{R}(Y)^{\perp_{M}} \rightarrow \mathcal{R}(Y)^{\perp_{M}},
$$

with $P_{\mathcal{R}(Y)^{\perp M_{1}}}=I-Y H_{1}^{-1} C_{1}^{T}$ and $H_{1}=C_{1}^{T} Y$. The inverse of this preconditioner (as a mapping from $\mathcal{R}(Y)^{\perp_{M}}$ onto itself) is 
Algorithm 1. Preconditioned conjugate gradient method for $(A-\sigma M) \mathbf{x}=M \mathbf{y}$.
Initialization:
(i) $\mathbf{r}_{0}:=M \mathbf{y}-(A-\sigma M) \mathbf{x}_{0}, \quad\left(C^{T} \mathbf{y}=\mathbf{0}\right.$ and, in general, $\left.\mathbf{x}_{0}=\mathbf{0}\right)$,
(ii) $\mathbf{z}_{0}:=\left(I-Y H^{-1} C^{T}\right)\left(A_{1}-\sigma_{1} M_{1}\right)^{-1} \mathbf{r}_{0}$,
(iii) $\mathbf{p}_{0}:=\mathbf{z}_{0}, \quad j:=0$.
Iteration: until convergence $\left(\left\|\mathbf{r}_{j}\right\|=\left\|M \mathbf{y}-(A-\sigma M) \mathbf{x}_{j}\right\|<\right.$ tol $\left.\| \mathbf{r}_{0}\right)$ do
(iv) $\alpha_{j}:=\left(\mathbf{z}_{j}^{T} \mathbf{r}_{j}\right) /\left[\mathbf{p}_{j}^{T}(A-\sigma M) \mathbf{p}_{j}\right]$,
(v) $\mathbf{x}_{j+1}:=\mathbf{x}_{j}+\alpha_{j} \mathbf{p}_{j}$,
(vi) $\mathbf{r}_{j+1}:=\mathbf{r}_{j}-\alpha_{j}(A-\sigma M) \mathbf{p}_{j}$,
(vii) $\mathbf{z}_{j+1}:=\left(I-Y H^{-1} C^{T}\right)\left(A_{1}-\sigma_{1} M_{1}\right)^{-1} \mathbf{r}_{j+1}$,
(viii) $\beta_{j}:=\left(\mathbf{z}_{j+1}^{T} \mathbf{r}_{j+1}\right) /\left(\mathbf{z}_{j}^{T} \mathbf{r}_{j}\right)$,
(ix) $\mathbf{p}_{j+1}:=\mathbf{z}_{j+1}+\beta_{j} \mathbf{p}_{j}$,
(x) $j:=j+1$.

$$
\begin{aligned}
P_{\mathcal{R}(Y)^{\perp_{M}}}\left(A_{1}\right. & \left.-\sigma_{1} M_{1}\right)^{-1} M \\
& =\left(I-Y H^{-1} C^{T}\right)\left(A_{1}-\sigma_{1} M_{1}\right)^{-1} M .
\end{aligned}
$$

The preconditioned conjugate gradient method for solving (15) with preconditioner (22) is displayed in Algorithm 1.

Notice that the inner products with respect to $M$ have disappeared. Operations with $M$ occur in the computation of the residuals and implicitly in the $M$-orthogonal projector. In Algorithm 1 the various iterates satisfy

$$
Y^{T} \mathbf{r}_{j}=C^{T} \mathbf{z}_{j}=C^{T} \mathbf{p}_{j}=C^{T} \mathbf{x}_{j}=\mathbf{0} \text { for all } j,
$$

such that the approximation $\mathbf{x}_{j}$ is in the correct space whenever we stop the iteration. Step (vii) in Algorithm 1 consumes most of the execution time. Two systems of equations have to be solved (1) with $A_{1}-\sigma_{1} M_{1}$ and (2) with $H$ inside the projector. In fact, we use a direct solver for the $(1,1)$ block $A_{11}-\sigma_{1} M_{11}$ and execute just one step of the Jacobi or symmetric Gauss-Seidel iteration for approximating the inverse of $A_{22}-\sigma_{1} M_{22}$; see Bank [31]. For solving Poisson's equation with $H$ we use a direct method as well.

\section{SOLVING THE CONSTRAINED SYSTEM OF EQUATION: THE AUGMENTED SYSTEM APPROACH}

An alternative way to write (15) is as an augmented linear system,

$$
\left[\begin{array}{cc}
A-\sigma M & C \\
C^{T} & O
\end{array}\right]\left[\begin{array}{l}
\mathbf{x}^{\prime} \\
\mathbf{x}^{\prime \prime}
\end{array}\right]=\left[\begin{array}{c}
M \mathbf{y} \\
\mathbf{0}
\end{array}\right] .
$$

If $A-\sigma M$ is nonsingular then the block LU factorization of the $2 \times 2$ block matrix in (24) immediately shows that $\mathbf{x}^{\prime \prime}=\mathbf{0}$. Therefore, the first component $\mathbf{x}^{\prime}$ of the solution $\mathbf{x}$ of (24) satisfies $(A-\sigma M) \mathbf{x}^{\prime}=M \mathbf{y}$ by the first equation and the constraints by the second equation.

The symmetric indefinite matrix in Eq. (24) has order $n+m$. It has $n$ positive and $m$ negative eigenvalues. Solving (24) instead of (20) has the advantage that there are no (explicit) constraints to be taken into account. There are, however, more unknowns and quite an accurate solution is needed to satisfy the constraints.

We considered preconditioners of the form

$$
\left[\begin{array}{cc}
A_{1}-\sigma_{1} M_{1} & C \\
C^{T} & O
\end{array}\right]
$$

when solving (24) by the preconditioned conjugate gradient method. $A_{1}$ and $M_{1}$ have been defined in (21). It turned out that this preconditioner leads to an algorithm that is very similar to the one of the straightforward approach. The difference is that the projector $I-$ $Y H^{-1} C^{T}$ is replaced by the much more complicated $I-$ $K_{1}^{-1} C\left(C^{T} K_{1}^{-1} C\right)^{-1} C^{T} K_{1}^{-1}$.

We therefore tried out preconditioners of the form

$$
\left[\begin{array}{cc}
K_{1} & C_{1} \\
C_{1}^{T} & O
\end{array}\right], \quad K_{1}=A_{1}-\sigma_{1} M_{1}, \quad C_{1}=M_{1} Y .
$$

We can easily transcribe Algorithm 1 into a solver for (24). Each vector in the algorithm now has two components, the first being in $\mathbb{R}^{n}$, the second in $\mathbb{R}^{m}$; see Algorithm 2. If $\mathbf{x}_{0}$ is chosen such that $C^{T} \mathbf{x}_{0}^{\prime}=\mathbf{0}$ then traversing stepwise through Algorithm 2 one sees that

$$
\mathbf{z}_{j}^{\prime \prime}=\mathbf{p}_{j}^{\prime \prime}=\mathbf{x}_{j}^{\prime \prime}=\mathbf{0} \text { for all } j,
$$

provided that $\sigma=\sigma_{1}$, which we assume henceforth. To verify (27) we observe that initially $C^{T} \mathbf{y}=C^{T} \mathbf{x}_{0}^{\prime}=\mathbf{0}$ such that $Y^{T} \mathbf{r}_{0}^{\prime}=\mathbf{0}$ and $\mathbf{r}_{0}^{\prime \prime}=\mathbf{0}$. Let us assume that (27) holds for all indices up to $j$. From step (v) in Algorithm 2 we see that $\mathbf{x}_{j+1}^{\prime \prime}=\mathbf{0}$. From step (vi) we have

$$
\begin{aligned}
Y^{T} \mathbf{r}_{j+1}^{\prime} & =Y^{T} \mathbf{r}_{j}^{\prime}-\alpha_{j}\left(-\sigma Y^{T} M\right) \mathbf{p}_{j}^{\prime} \\
& =Y^{T} \mathbf{r}_{j}^{\prime}+\alpha_{j} \sigma C^{T} \mathbf{p}_{j}^{\prime}, \\
\mathbf{r}_{j+1}^{\prime \prime} & =\mathbf{r}_{j}^{\prime \prime}-\alpha_{j} C^{T} \mathbf{p}_{j}^{\prime} .
\end{aligned}
$$

The second component of the solution of the equation

$$
\begin{aligned}
{\left[\begin{array}{c}
\mathbf{z}_{j+1}^{\prime} \\
\mathbf{z}_{j+1}^{\prime \prime}
\end{array}\right] } & =\left[\begin{array}{cc}
K_{1} & C_{1} \\
C_{1}^{T} & O
\end{array}\right]^{-1}\left[\begin{array}{c}
\mathbf{r}_{j+1}^{\prime} \\
\mathbf{r}_{j+1}^{\prime \prime}
\end{array}\right] \\
& =\left[\begin{array}{ccc}
K_{1}^{-1}+(1 / \sigma) Y H_{1}^{-1} Y^{T} & Y H_{1}^{-1} \\
& H_{1}^{-1} Y^{T} & \sigma H_{1}^{-1}
\end{array}\right]\left[\begin{array}{c}
\mathbf{r}_{j+1}^{\prime} \\
\mathbf{r}_{j+1}^{\prime \prime}
\end{array}\right]
\end{aligned}
$$


Algorithm 2. Preconditioned conjugate gradient method for the augmented linear system.

Initialization:

(i) $\mathbf{r}_{0}:=\left[\begin{array}{c}M \mathbf{y}-(A-\sigma M) \mathbf{x}_{0}^{\prime}-C \mathbf{x}_{0}^{\prime \prime} \\ -C^{T} \mathbf{x}_{0}^{\prime} \\ \left(C^{T} \mathbf{y}=C^{T} \mathbf{x}_{0}^{\prime}=\mathbf{0}, \mathbf{x}_{0}^{\prime \prime}=\mathbf{0}\right) .\end{array}\right.$

(ii) Solve $\left[\begin{array}{cc}A_{1}-\sigma_{1} M_{1} & C_{1} \\ C_{1}^{T} & O\end{array}\right]\left[\begin{array}{l}\mathbf{z}_{0}^{\prime} \\ \mathbf{z}_{0}^{\prime \prime}\end{array}\right]=\left[\begin{array}{l}\mathbf{r}_{0}^{\prime} \\ \mathbf{r}_{0}^{\prime \prime}\end{array}\right]$,

(iii) $\mathbf{p}_{0}:=\mathbf{z}_{0}, \quad j:=0$.

Iteration: until convergence $\left(\left\|\mathbf{r}_{j}\right\|<\operatorname{tol}\left\|\mathbf{r}_{0}\right\|\right)$ do

(iv) $\alpha_{j}:=\left(\mathbf{z}_{j}^{T} \mathbf{r}_{j}\right) /\left(\mathbf{p}_{j}^{T}\left[\begin{array}{cc}A-\sigma M & C \\ C^{T} & O\end{array}\right] \mathbf{p}_{j}\right)$,

(v) $\mathbf{x}_{j+1}:=\mathbf{x}_{j}+\alpha_{j} \mathbf{p}_{j}$,

(vi) $\mathbf{r}_{j+1}:=\mathbf{r}_{j}-\alpha_{j}\left[\begin{array}{cc}A-\sigma M & C \\ C^{T} & O\end{array}\right] \mathbf{p}_{j}$.

(vii) Solve $\left[\begin{array}{cc}A_{1}-\sigma_{1} M_{1} & C_{1} \\ C_{1}^{T} & O\end{array}\right]\left[\begin{array}{l}\mathbf{z}_{j+1}^{\prime} \\ \mathbf{z}_{j+1}^{\prime \prime}\end{array}\right]=\left[\begin{array}{c}\mathbf{r}_{j+1}^{\prime} \\ \mathbf{r}_{j+1}^{\prime \prime}\end{array}\right]$,

(viii) $\beta_{j}:=\left(\mathbf{z}_{j+1}^{T} \mathbf{r}_{j+1}\right) /\left(\mathbf{z}_{j}^{T} \mathbf{r}_{j}\right)$,

(ix) $\mathbf{p}_{j+1}:=\mathbf{z}_{j+1}+\beta_{j} \mathbf{p}_{j}$,

(x) $j:=j+1$.

in step (vii) of the algorithm is

$$
\begin{aligned}
\mathbf{z}_{j+1}^{\prime \prime} & =H_{1}^{-1}\left(Y^{T} \mathbf{r}_{j+1}^{\prime}+\sigma \mathbf{r}_{j+1}^{\prime \prime}\right) \\
& =H_{1}^{-1}\left(Y^{T} \mathbf{r}_{j}^{\prime}+\alpha_{j} \sigma C^{T} \mathbf{p}_{j}^{\prime}+\sigma \mathbf{r}_{j}^{\prime \prime}-\alpha_{j} \sigma C^{T} \mathbf{p}_{j}^{\prime}\right) \\
& =H_{1}^{-1}\left(Y^{T} \mathbf{r}_{j}^{\prime}+\sigma \mathbf{r}_{j}^{\prime \prime}\right) \\
& =\cdots=H_{1}^{-1}\left(Y^{T} \mathbf{r}_{0}^{\prime}+\sigma \mathbf{r}_{0}^{\prime \prime}\right)=\mathbf{0}
\end{aligned}
$$

Here, we have used that $K_{1}=A_{1}-\sigma M_{1}$ is nonsingular and that $K_{1} Y=-\sigma C_{1} \cdot \mathbf{p}_{j+1}^{\prime \prime}=\mathbf{0}$ follows from step (ix) in Algorithm 2.

The relations in (27) simplify the algorithm considerably. Only the $\mathbf{r}_{j}$ have a nonzero second component. It is used in step (vii) of the algorithm to compute $\mathbf{z}_{j}$, in fact, $\mathbf{z}_{j}^{\prime}$ as $\mathbf{z}_{j}^{\prime \prime}=\mathbf{0}$ as we have just seen. From (28) we get

$$
\begin{aligned}
\mathbf{z}_{j+1}^{\prime} & =K_{1}^{-1} \mathbf{r}_{j+1}^{\prime}+\frac{1}{\sigma} Y H_{1}^{-1}\left(Y^{T} \mathbf{r}_{j+1}^{\prime}+\sigma \mathbf{r}_{j+1}^{\prime \prime}\right) \\
& =K_{1}^{-1} \mathbf{r}_{j+1}^{\prime} .
\end{aligned}
$$

So, actually, $\mathbf{r}_{j}^{\prime \prime}$ is never used and thus does not have to be computed at all. It can be recovered anytime as $\mathbf{r}_{j}^{\prime \prime}=$ $(-1 / \sigma) Y^{T} \mathbf{r}_{j}^{\prime}$.

Taking all the above simplifications into account, Algorithm 2 with preconditioner (26) becomes Algorithm 3.

Notice that Algorithm 3 differs from Algorithm 1 in that the projectors $I-Y H^{-1} C^{T}$ are omitted. In fact, in Algorithm 3 plain pcg is applied to the consistent but indefinite system of equations $(A-\sigma M) \mathbf{x}=M \mathbf{y}$. It is not yet clear to us if this algorithm is stable at all. Certainly, the constraints $C^{T} \mathbf{x}_{j+1}^{\prime}=\mathbf{0}$ are not satisfied before convergence. If we solve systems as in the Jacobi-Davidson algorithm with only low accuracy then we enforce the constraints explicitly at the end of the iteration.
Algorithm 3. Preconditioned conjugate gradient method for the augmented linear system after simplifications.

Initialization:
(i) $\mathbf{r}_{0}^{\prime}:=M \mathbf{y}-(A-\sigma M) \mathbf{x}_{0}^{\prime}, \quad\left(C^{T} \mathbf{y}=C^{T} \mathbf{x}_{0}^{\prime}=\mathbf{0}\right)$,
(ii) $\mathbf{z}_{0}^{\prime}:=\left(A_{1}-\sigma M_{1}\right)^{-1} \mathbf{r}_{0}^{\prime}$,
(iii) $\mathbf{p}_{0}^{\prime}:=\mathbf{z}_{0}^{\prime}, \quad j:=0$.

Iteration: until convergence $\left(\left\|\mathbf{r}_{j}\right\|<\operatorname{tol}\left\|\mathbf{r}_{0}\right\|\right)$ do
(iv) $\alpha_{j}:=\left(\mathbf{z}_{j}^{\prime T} \mathbf{r}_{j}^{\prime}\right) /\left[\mathbf{p}_{j}^{\prime T}(A-\sigma M) \mathbf{p}_{j}^{\prime}\right]$,
(v) $\mathbf{x}_{j+1}^{\prime}:=\mathbf{x}_{j}^{\prime}+\alpha_{j} \mathbf{p}_{j}^{\prime}$,
(vi) $\mathbf{r}_{j+1}^{\prime}:=\mathbf{r}_{j}^{\prime}-\alpha_{j}(A-\sigma M) \mathbf{p}_{j}^{\prime}$,
(vii) $\mathbf{z}_{j+1}^{\prime}:=\left(A_{1}-\sigma M_{1}\right)^{-1} \mathbf{r}_{j+1}^{\prime}$,
(viii) $\beta_{j}:=\left(\mathbf{z}_{j+1}^{\prime T} \mathbf{r}_{j+1}^{\prime}\right) /\left(\mathbf{z}_{j}^{\prime T} \mathbf{r}_{j}^{\prime}\right)$,
(ix) $\mathbf{p}_{j+1}^{\prime}:=\mathbf{z}_{j+1}^{\prime}+\beta_{j} \mathbf{p}_{j}^{\prime}$,
(x) $j:=j+1$.

\section{NUMERICAL EXPERIMENTS}

We performed a few numerical experiments computing the ten lowest eigenvalues and corresponding eigenvectors of a model of the future design of the copper cavity; cf. Fig. 1. The finite element discretization is still quite coarse and symmetries of the geometry have not been taken into account for these tests of the solution methods. We computed the eigenpairs to an accuracy $\varepsilon=$ $10^{-4}$, which means that we accepted an eigenpair $(\lambda, \mathbf{x})$ if $\|A \mathbf{x}-\lambda M \mathbf{x}\|_{2}<\varepsilon$. As $\left\|M^{-1}\right\|_{2} \approx 10^{5}$ and the gap between the eigenvalues is about five, this guarantees that all the interesting modes with frequencies in the range of $50-500 \mathrm{MHz}$ are defined to an accuracy better than $50 \mathrm{kHz}$ [12]. We used three different discretizations, a coarse discretization with only $n=1880$ degrees of freedom, a fine discretization with 27088 , and an even finer discretization with 45040 degrees of freedom. The corresponding values for $n_{1}$ and $m_{1}$ (cf. (8)] are given in Tables I and II.

We combined the implicitly restarted Lanczos algorithm and the Jacobi-Davidson algorithm with the straightforward ("projection") or modified augmented approach for solving (15). With IRL we solved the positive definite systems of equations in the straightforward approach with the preconditioned conjugate gradient method. With JD the shifts vary dynamically and can get inside the convex hull of the spectrum. That is why we used MINRES as our system solver [29]. The systems of equations in the augmented system where the preconditioner is indefinite were solved with a special variant of QMR algorithm [32]. In either case, we set $\sigma_{1}=\sigma$ to be a value slightly below the lowest positive eigenvalue. When solving (15), we employed no diagonal or hierarchical basis preconditioning. The last was combined with two different treatments of the $A_{22}$ block. Either we performed one step of damped Jacobi (damping factor $2 / 3$ ) or one step of symmetric Gauss-Seidel. The results for IRL are summarized in Table I, those for JD in Table II. The number $i t_{\text {int }}$ provides the average number of inner iterations, i.e., the steps until convergence of 
TABLE I. Implicitly restarted Lanczos (ARPACK) results for the copper cavity. Times are in seconds.

\begin{tabular}{|c|c|c|c|c|c|}
\hline \multicolumn{6}{|c|}{ Small problem size: $n=1880, n_{1}=300, m_{1}=27$} \\
\hline Method, preconditioner & $i t_{\mathrm{int}}$ & $i t_{\text {out }}$ & $t_{\mathrm{tot}}$ & $t_{\mathrm{eig}}$ & $t_{11}$ \\
\hline Projection, none & 1018 & 70 & 334 & 333 & \\
\hline Projection, diagonal & 166 & 70 & 57 & 56 & \\
\hline Projection, hierarchical/damped Jacobi & 145 & 70 & 69 & 68 & 15 \\
\hline Projection, hierarchical/symmetric Gauss-Seidel & 72 & 70 & 51 & 50 & 9 \\
\hline Augmented, hierarchical/damped Jacobi & 153 & 70 & 39 & 38 & 15 \\
\hline Augmented, hierarchical/symmetric Gauss-Seidel & 78 & 70 & 33 & 32 & 8 \\
\hline \multicolumn{6}{|c|}{ Medium problem size: $n=27088, n_{1}=4688, m_{1}=507$} \\
\hline Method, preconditioner & $i t_{\text {int }}$ & $i t_{\text {out }}$ & $t_{\text {tot }}$ & $t_{\text {eig }}$ & $t_{11}$ \\
\hline Projection, none & 842 & 34 & 8744 & 8661 & \\
\hline Projection, diagonal & 244 & 34 & 2632 & 2551 & \\
\hline Projection, hierarchical/damped Jacobi & 98 & 34 & 1579 & 1493 & 433 \\
\hline Projection, hierarchical/symmetric Gauss-Seidel & 50 & 34 & 937 & 851 & 218 \\
\hline Augmented, hierarchical/damped Jacobi & 100 & 34 & 768 & 675 & 410 \\
\hline Augmented, hierarchical/symmetric Gauss-Seidel & 52 & 34 & 530 & 438 & 209 \\
\hline \multicolumn{6}{|c|}{ Large problem size: $n=45040, n_{1}=7824, m_{1}=867$} \\
\hline Method, preconditioner & $i t_{\text {int }}$ & $i t_{\mathrm{out}}$ & $t_{\mathrm{tot}}$ & $t_{\text {eig }}$ & $t_{11}$ \\
\hline Projection, none & 667 & 34 & 12517 & 12306 & \\
\hline Projection, diagonal & 283 & 34 & 5390 & 5179 & \\
\hline Projection, hierarchical/damped Jacobi & 79 & 34 & 2459 & 2234 & 734 \\
\hline Projection, hierarchical/symmetric Gauss-Seidel & 41 & 34 & 1569 & 1342 & 389 \\
\hline Augmented, hierarchical/damped Jacobi & 80 & 34 & 1324 & 1080 & 701 \\
\hline Augmented, hierarchical/symmetric Gauss-Seidel & 42 & 34 & 955 & 712 & 365 \\
\hline
\end{tabular}

the linear solver. As systems are solved to much higher accuracy with IRL (to establish the Lanczos three-term recurrence [28]) the iteration count $i t_{\text {int }}$ is much bigger in Table I than in Table II. In fact, with JD, the convergence tolerance of the inner iteration is initially very weak and becomes as stringent as with IRL as the outer iteration converges [9]. In contrast, the number of outer iterations $i t_{\text {out }}$, i.e., the number of systems that are solved, is smaller with IRL. In both IRL and JD we let the dimension of the trial space vary between 10 and 25 .

$t_{\text {tot }}$ gives the overall solution time, including grid handling, matrix assembly, eigensolver, etc., while $t_{\text {eig }}$ is the time spent for solving the eigensystem only. Finally, $t_{11}$ gives the time that is spent for solving (forward/backward substitution) the upper diagonal $(1,1)$ block by a direct solver. $t_{\text {eig }}$ includes $t_{11}$. These two numbers are the execution times of interest. Our discussion is based on them.

The numerical experiments were run in sequential mode on a Sun Enterprise 3500 with six $336 \mathrm{MHz}$ UltraSparc processors and 3 GB main memory running the Solaris 2.6 operating system. All times are user time in seconds.

In all examples, the Jacobi-Davidson algorithm is the faster eigensolver than the implicitly restarted Lanczos algorithm. This is due to the fact that IRL executes many more steps in the inner iteration as the Lanczos vectors have to be computed to high accuracy.

Higher sophistication in the preconditioner decreases not only the iteration counts but also the execution times.
This becomes more prominent as the problem size increases. This is in contrast to the experiments that we conducted with node elements in the box-shaped cavity [33]. The analysis of the hierarchical basis preconditioner states that $i t_{\text {int }}$ should not increase with the problem size. Here it even decreases. Further, in accordance with theory, the Gauss-Seidel smoothing halves the iteration count of Jacobi smoothing [31].

When comparing the straightforward with the (modified) augmented system approach, IRL and JD behave quite differently. With IRL we observe a big gain of almost 50\% when employing the augmented system approach combined with damped Jacobi smoothing. The gain is smaller but still high with symmetric Gauss-Seidel. The average number of inner iterations differs only slightly in the two approaches.

With Jacobi-Davidson the gain with the augmented system is smaller but still considerable with either damped Jacobi or symmetric Gauss-Seidel smoothing. However, as the average number of inner iterations grows, the reduced cost per iteration by saving the projector is to a large extent compensated by a higher number of iterations.

It is worth noting that the time $t_{11}$ for solving the $(1,1)$ block takes a considerable fraction of the solution of the eigenvalue problem. $t_{11}$ is about $20 \%$ of $t_{\text {eig }}$ in the medium sized problems and about $25 \%$ in the large problems. With the augmented system of equation this portion is with IRL and Jacobi smoothing even above 50\%. In this case, the 
TABLE II. Jacobi-Davidson results for the copper cavity. Times are in seconds.

\begin{tabular}{|c|c|c|c|c|c|}
\hline \multicolumn{6}{|c|}{ Small problem size: $n=1880, n_{1}=300, m_{1}=27$} \\
\hline Method, preconditioner & $i t_{\text {int }}$ & $i t_{\text {out }}$ & $t_{\text {tot }}$ & $t_{\text {eig }}$ & $t_{11}$ \\
\hline Projection, none & 163 & 129 & 203 & 202 & \\
\hline Projection, diagonal & 29 & 106 & 33 & 32 & \\
\hline Projection, hierarchical/Jacobi & 28 & 110 & 36 & 35 & 5 \\
\hline Projection, hierarchical/symmetric Gauss-Seidel & 13 & 101 & 20 & 20 & 2 \\
\hline Augmented, hierarchical/Jacobi & 43 & 106 & 28 & 28 & 8 \\
\hline Augmented, hierarchical/symmetric Gauss-Seidel & 24 & 98 & 20 & 19 & 4 \\
\hline \multicolumn{6}{|c|}{ Medium problem size: $n=27088, n_{1}=4688, m_{1}=507$} \\
\hline Method, preconditioner & $i t_{\text {int }}$ & $i t_{\text {out }}$ & $t_{\mathrm{tot}}$ & $t_{\text {eig }}$ & $t_{11}$ \\
\hline Projection, none & 73 & 95 & 2889 & 2808 & \\
\hline Projection, diagonal & 17 & 84 & 752 & 671 & \\
\hline Projection, hierarchical/Jacobi & 11 & 80 & 664 & 579 & 131 \\
\hline Projection, hierarchical/symmetric Gauss-Seidel & 5 & 79 & 466 & 381 & 75 \\
\hline Augmented, hierarchical/Jacobi & 15 & 74 & 478 & 385 & 163 \\
\hline Augmented, hierarchical/symmetric Gauss-Seidel & 8 & 74 & 372 & 279 & 97 \\
\hline \multicolumn{6}{|c|}{ Large problem size: $n=45040, n_{1}=7824, m_{1}=867$} \\
\hline Method, preconditioner & $i t_{\mathrm{int}}$ & $i t_{\text {out }}$ & $t_{\mathrm{tot}}$ & $t_{\mathrm{eig}}$ & $t_{11}$ \\
\hline Projection, none & 55 & 89 & 3887 & 3680 & \\
\hline Projection, diagonal & 19 & 86 & 1501 & 1289 & \\
\hline Projection, hierarchical/Jacobi & 8 & 82 & 1122 & 900 & 237 \\
\hline Projection, hierarchical/symmetric Gauss-Seidel & 4 & 76 & 816 & 593 & 137 \\
\hline Augmented, hierarchical/Jacobi & 12 & 75 & 907 & 659 & 306 \\
\hline Augmented, hierarchical/symmetric Gauss-Seidel & 7 & 74 & 721 & 474 & 184 \\
\hline
\end{tabular}

direct solver appears to not yet having been implemented optimally. In any case, the present two-level preconditioner will not suffice for larger problem sizes. The direct solution of the system of equations corresponding to the $(1,1)$ block $A_{11}-\sigma M_{11}$ will have to be replaced by a more sophisticated method such as, e.g., algebraic multigrid.

\section{CONCLUSIONS}

We have investigated algorithms for solving the eigenvalue problem occurring in the design of resonant cavities. The governing Maxwell equations have been discretized in a mixed mode finite element approach using quadratic Nédélec edge elements for the electric field intensity and quadratic node-based elements for the Lagrange multipliers. This approach naturally enforces divergence-free fields and avoids spurious modes. The finite element spaces for the model problems used to study the algorithm had dimensions up to 45040 . The resulting symmetric generalized matrix eigenvalue problems have been solved by two methods: the Jacobi-Davidson and the implicitly restarted Lanczos algorithms. In both methods, a shift-and-invert spectral transformation has been applied. The such introduced symmetric indefinite systems of equations have been solved iteratively by a Krylov subspace method. The most effective preconditioners with respect to execution time and iteration numbers were hierarchical basis preconditioners. The average number of iterations to solve these systems not only stayed constant but decreased with increasing problem size.

The JD algorithm clearly outperforms IRL. The execution times of the latter are about twice as long due to the high accuracy requirement imposed by the construction of a correct three-term recurrence.

The symmetric indefinite systems of equations have been solved in two ways. First, by projecting in the subspace where the system is positive definite, and, second, by augmenting the system, i.e., by introducing constraints and Lagrange multipliers. With the first we obtained very satisfactory results. The latter still improved the timings essentially with IRL but only slightly with JD. In other experiments where we computed the eigenpairs to higher accuracy the projection method was clearly superior. The augmented systems approach is not yet completely understood with regard to its convergence behavior and stability. Further investigations in this direction are required.

[1] J. D. Jackson, Classical Electrodynamics (Wiley, New York, 1975), 2nd ed.

[2] F. Kikuchi, Comput. Methods Appl. Mech. Eng. 64, 509 (1987).

[3] J. Jin, The Finite Element Method in Electromagnetics (Wiley, New York, 1993).

[4] J. C. Nédélec, Numer. Math. 35, 315 (1980).

[5] J. P. Webb, IEEE Trans. Magn. 29, 1460 (1993).

[6] G. Mur, IEEE Trans. Magn. 30, 3552 (1994). 
[7] T. Weiland, Part. Accel. 15, 245 (1984).

[8] T. Weiland, Part. Accel. 17, 227 (1985).

[9] P. Arbenz and R. Geus, Numer. Lin. Alg. Appl. 6, 3 (1999).

[10] D.C. Sorensen, SIAM J. Matrix Anal. Appl. 13, 357 (1992).

[11] G. L. G. Sleijpen and H. A. van der Vorst, SIAM J. Matrix Anal. Appl. 17, 401 (1995).

[12] B. N. Parlett, The Symmetric Eigenvalue Problem (Prentice Hall, Englewood Cliffs, NJ, 1980) (republished by SIAM, Philadelphia, 1998).

[13] R. Grimes, J. G. Lewis, and H. Simon, SIAM J. Matrix Anal. Appl. 15, 228 (1994).

[14] P. G. Ciarlet, in The Finite Element Method for Elliptic Problems, Studies in Mathematics and its Applications Vol. 4 (North-Holland, Amsterdam, 1978).

[15] A. Bossavit, Computational Electromagnetism (Academic, San Diego, 1998).

[16] V. Girault and P.-A. Raviart, in Finite Element Methods for the Navier-Stokes Equations, Springer Series in Computational Mathematics Vol. 5 (Springer, Berlin, 1986).

[17] P. P. Silvester and R. L. Ferrari, Finite Elements for Electrical Engineers (Cambridge University, Cambridge, England, 1996), 3rd ed.

[18] J. S. Savage and A. F. Peterson, IEEE Trans. Microwave Theory Tech. 44, 874 (1996).

[19] R. Gruber and J. Rappaz, Finite Element Methods in Linear Ideal Magnetohydrodynamics (Springer, Berlin, 1985).

[20] H. Rutishauser, Numer. Math. 16, 205 (1970), reprinted in Linear Algebra, edited by J. H. Wilkinson and C. Reinsch (Springer, Berlin, 1971), pp. 284-301.
[21] D. Calvetti, L. Reichel, and D.C. Sorensen, Electron. Trans. Numer. Anal. 2, 1 (1994).

[22] J. Baglama, D. Calvetti, and L. Reichel, BIT 36, 400 (1996).

[23] G. L. G. Sleijpen, A. G. L. Booten, D. R. Fokkema, and H. A. van der Vorst, BIT 36, 5951996.

[24] D. R. Fokkema, G. L. G. Sleijpen, and H. A. van der Vorst, SIAM J. Sci. Comput. 20, 94 (1998).

[25] D. S. Scott, SIAM J. Sci. Stat. Comput. 3, 68 (1982).

[26] T. Ericsson, in Matrix Pencils, edited by B. Kågström and A. Ruhe, Lecture Notes in Mathematics Vol. 973 (Springer, Berlin, 1983), pp. 177-188.

[27] B. Nour-Omid, B. N. Parlett, T. Ericsson, and P. S. Jensen, Math. Comput. 48, 663 (1987).

[28] R. B. Lehoucq, D. C. Sorensen, and C. Yang, ARPACK Users' Guide: Solution of Large-Scale Eigenvalue Problems by Implicitly Restarted Arnoldi Methods (SIAM, Philadelphia, 1998). (The software and this manual are available at URL http://www.caam.rice.edu/software/ARPACK/).

[29] Y. Saad, Iterative Methods for Sparse Linear Systems (PWS Publishing Company, Boston, 1996).

[30] C. C. Paige, B. N. Parlett, and H. A. van der Vorst, Numer. Linear Alg. Appl. 2, 115 (1995).

[31] R. E. Bank, Acta Numer. 5, 1 (1996).

[32] R. Freund and N. M. Nachtigal, Appl. Numer. Math. 19, 319 (1995).

[33] P. Arbenz and S. Adam, in Proceedings of the International Computational Accelerator Physics Conference (ICAP'98), Monterey, CA, 1998, http://www.inf.ethz.ch/ arbenz/ICAP98.ps.gz. 